\title{
Controlled Spacecraft Re-Entry of a Drag De-Orbit Device (D3)
}

\author{
Tanya Martin, Sanny Omar, Riccardo Bevilacqua \\ University of Florida
}

Faculty mentor: Riccardo Bevilacqua, Department of Mechanical and Aerospace Engineering

\begin{abstract}
For spacecraft containing components that survive re-entry, it is important to de-orbit the satellite over a non-populated area. Because most CubeSats (cube satellites that conform to the CubeSat form factor) do not have their own propulsion systems and cannot perform a de-orbit burn, aerodynamic drag modulation presents an attractive solution to re-enter the satellite at the desired location. The University of Florida Advanced Autonomous Multiple Spacecraft (ADAMUS) lab has developed a drag de-orbit device (D3) for CubeSats, which are affordable systems for demonstrating attitude and orbit control. The device consists of four retractable tape-spring booms that are designed and manufactured to validate the targeted re-entry of a CubeSat in low Earth orbit. By modulating the D3 drag area, orbital maneuvering and controlled re-entry can be performed. This paper outlines the functional and vibration testing procedures and results for the completed device. The complete drag device was taken to NASA Ames Research Center and subjected to random vibrations at 9.6 times the force of gravity. The device survived vibration testing with no obvious damage. After vibration testing, it was determined that no major mechanical design changes to the D3 will need to be made. Future work for this project includes assembling a final, flight-ready drag device that will be attached to a CubeSat for launch.

Keywords: aerodynamic drag, controlled re-entry, CubeSat, de-orbit, satellite, vibration
\end{abstract}

\section{Introduction}

The increasing number of space vehicles launched and abandoned in Low-Earth Orbit (LEO) present a concern of debris via accidental explosions as a result of collisions. From 1987 to 2007, the number of spacecraft launched into LEO has increased by $80 \%$, generating over 130 metric tons of spacecraft mass 0 . To mitigate the orbital debris issue, 0 has restricted the orbital lifetime of spacecraft by requiring LEO spacecraft to de-orbit within 25 years. Additionally, the probability of world-wide human casualty from a single re-entering structure must be less than 1 in 10,000 0. According to 0 cube satellites (CubeSats) are required to meet certain mass and volume requirements (up to $1.33 \mathrm{~kg}$ per $10 \mathrm{~cm}^{3}$ ), so most CubeSats do not contain their own propulsion systems that would enable re-entry into Earth's atmosphere via thrusters. Instead, modulating the aerodynamic drag of a spacecraft presents a viable solution to orbital debris mitigation requirements. While several teams have created and tested drag devices such as drag 
sails 00 and parachutes 0 , these devices are limited in their abilities such as how far they can retract, or if they can retract at all. So far, there has not been a successful controlled de-orbit of a small spacecraft into Earth's atmosphere using aerodynamic drag.

The University of Florida Advanced Autonomous Multiple Spacecraft (ADAMUS) Lab has developed a retractable drag-deorbit device (D3) 0 to be attached to a host CubeSat. It is capable of modulating the drag area of its CubeSat with four retractable tape-spring booms that are attached to four deployers. Combined with a targeting algorithm also developed by the ADAMUS lab 0, the D3 can be utilized for orbital maneuvering while maintaining passive 3-axis attitude stability. The goal of the D3 device is to present an affordable and reliable way to perform targeted re-entry of spacecraft, thus minimizing risk by avoiding collision with space debris and enabling LEO CubeSats to meet NASA orbital debris mitigation requirements. As part of the project, a complete D3 prototype was manufactured and assembled in-house. Functional tests were performed on the $\mathrm{D} 3$ to ensure that all electronic components produced desired results. To validate the structural integrity of the device, it was attached to a CubeSat frame and vibration tested. After passing vibration testing, the D3 device is believed to survive the conditions of launch and helps further prove a feasible way for re-entry of spacecraft. This paper gives an overview of the completed device and describes the testing procedures and results of vibration testing, as well as future plans for the course of the $\mathrm{D} 3$ project.

\section{Methodology}

\section{Drag De-Orbit Device (D3) and CubeSat Design Overview}

The D3 is made up of four retractable deployers with tape-spring booms arranged in a "dart" configuration on a base plate (Figure 1). Each boom is $3.7 \mathrm{~m}$ long by $4 \mathrm{~cm}$ wide and can be actuated independently with its own DC motor and encoder. An expanded view of one of the D3 deployers is shown in Figure 2. A minimum moment of inertia axis is created along two fullydeployed booms that are opposite to each other. The dart configuration of the booms enables the satellite to aerodynamically stabilize, with the long axis of the satellite aligned with the velocity vector. 

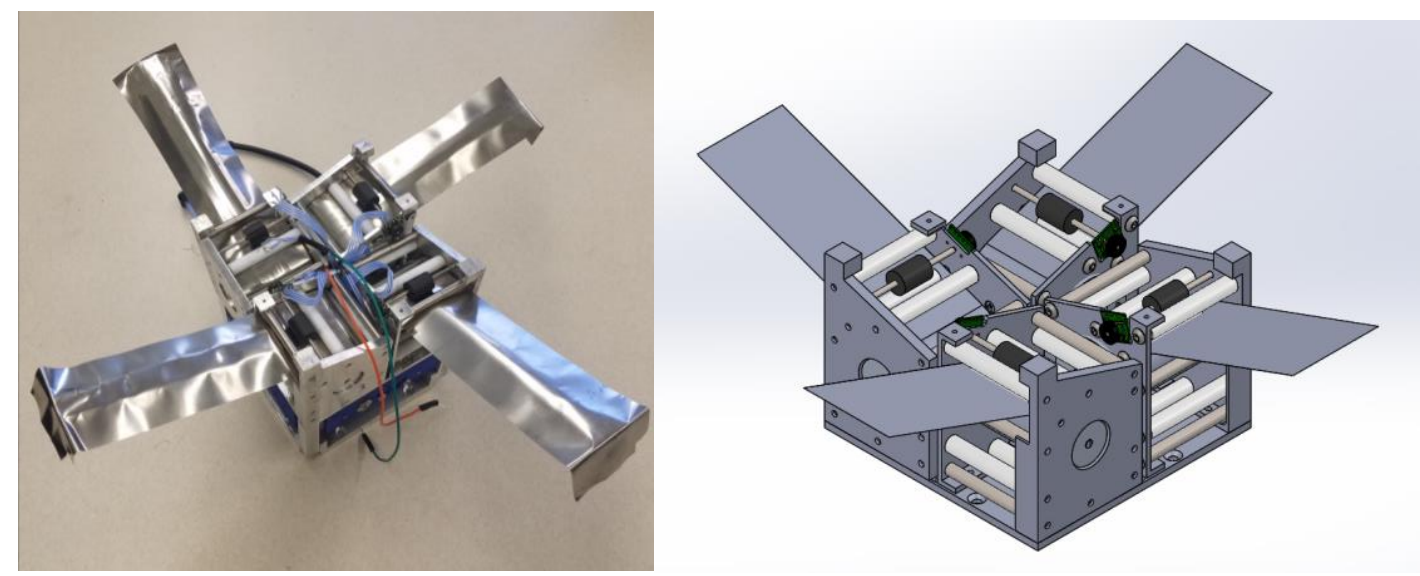

Figure 1. Prototype of the drag device (left) and its computer-aided design model (right). The aluminum and steel parts of the D3 device were manufactured in-house with a lathe, manual milling machine, and CNC router.

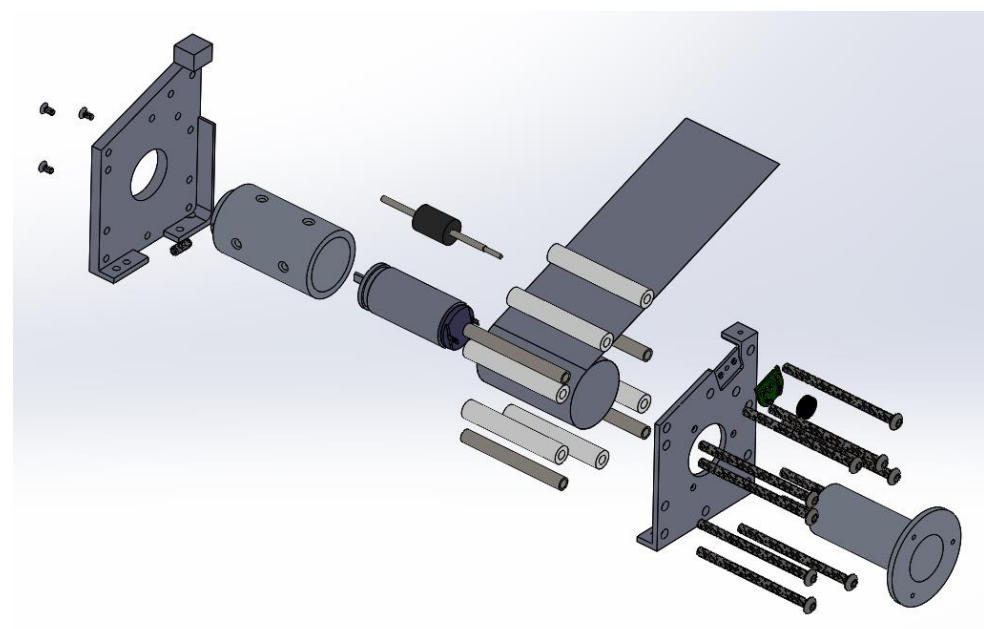

Figure 2. Expanded view of the D3 deployer assembly model.

The drag device is then attached to a host $1 \mathrm{U}\left(10 \mathrm{~cm}^{3}\right)$ CubeSat that contains five avionics boards, a GPS antenna, a deployable UHF turnstile communication antenna, and five custommade solar panels. The five avionics boards consist of a radio, a receiver, an EPS, a battery, and a D3 control board. The device, along with a custom-made adapter stage and brackets that hold three magnetorquers, all fit the volume and mass requirements of a $2 \mathrm{U}$ CubeSat (see Figure 3). The magnetorquers are made of copper wire and serve to dampen any attitude oscillations and increase the attitude stability of the satellite. 


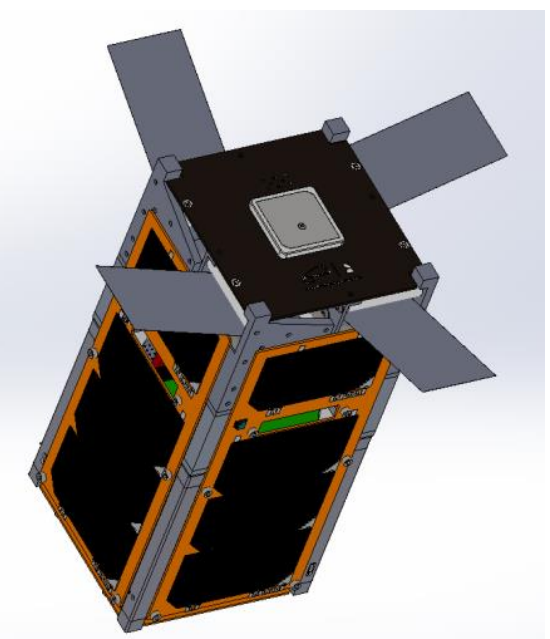

Figure 3. Satellite assembly model, which includes the D3 device, avionics boards, antenna, and solar panels.

\section{Vibration Testing Plans}

The purpose of vibration testing the D3 CubeSat is to simulate the random vibrations that the satellite will experience during launch. 0 outlines a set of General Environmental Verification Standards (GEVS) that serve as a baseline for demonstrating satisfactory hardware performance in their expected environments. When CubeSats are launched, they are placed in spring-loaded launchers that fully contain the satellite until they are ready to be released into LEO. The launcher that was available to use was a PPOD launcher as pictured in Figure 4, but on the real launch, the satellite will most likely be flying with a NanoRacks launcher. The satellite was not tested at GEVS levels, but rather the NanoRacks vibration levels (9.6 grms), which are slightly lower than those of GEVS. For the purposes of vibration testing, only the D3, the adapter stage, and 3D-printed solar panel and magnetorquer brackets have been assembled to the satellite (Figure 5). The actual solar panels and four of the avionics boards will be ordered from vendors in the future once a flight-ready device is made. The final avionics board is the D3 flight control board, which is to be designed and fabricated in the future. 


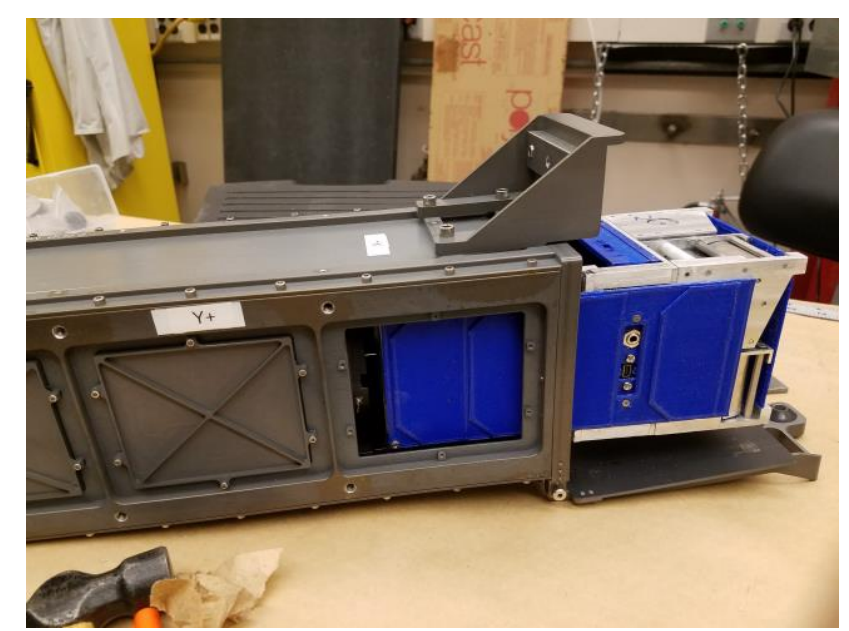

Figure 4. PPOD launcher with D3 CubeSat partially fitted inside.

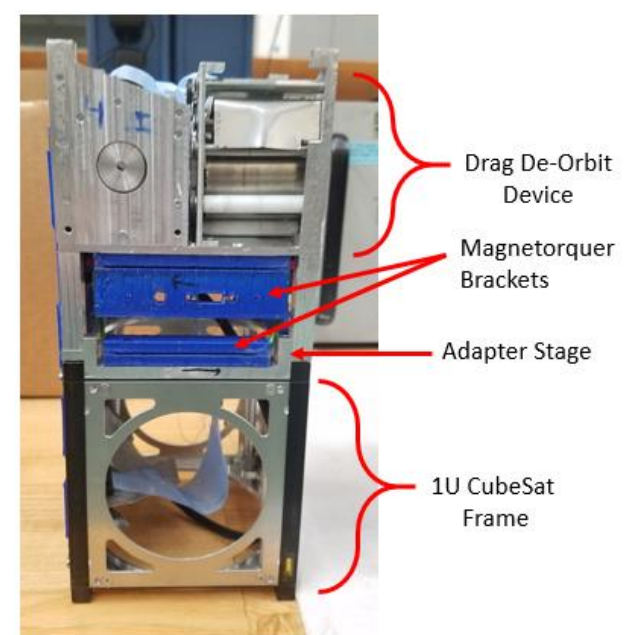

Figure 5. NanoRacks launcher with D3 CubeSat partially fitted inside.

The satellite prototype was vibration tested with a vibration table at NASA Ames Research Center in Mountain View, California (Figure 6). Before testing, a functional test was performed on the satellite to ensure that all four deployer motors and encoders work nominally. Also before testing, the satellite needed to be secured in the launcher. The outer dimensions of the prototype, however, prevented it from easily sliding in and out of the launcher. To get a better fit inside the launcher, the D3's outer deployer shells were filed down with a Dremel rotary tool and metal files (Figure 7). 


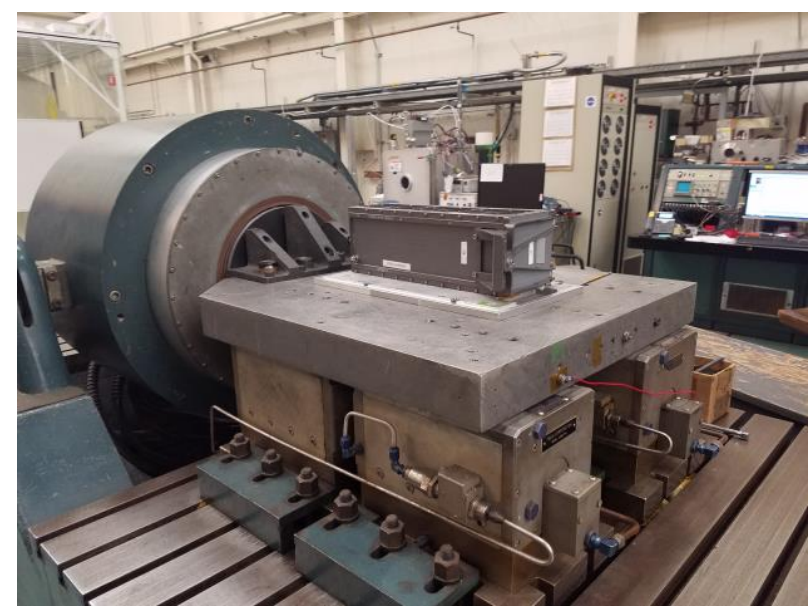

Figure 6. Vibration table at NASA Ames Research Center.

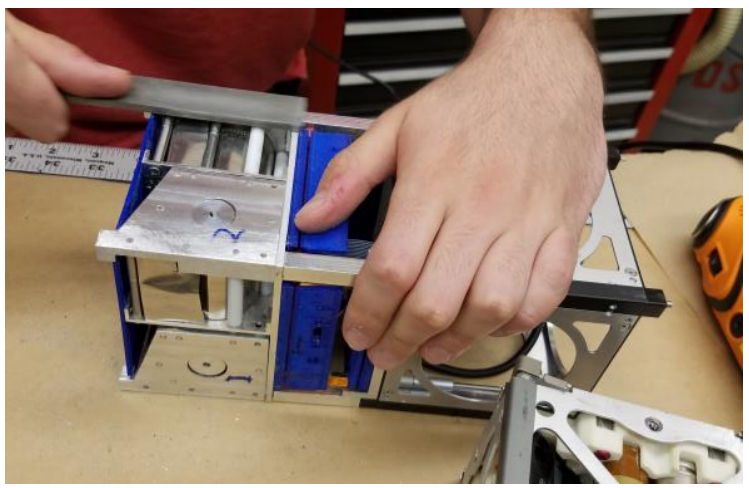

Figure 7. Filing down the outermost edges of the D3 device.

After being secured in the launcher, the launcher was attached to the vibration table via aluminum base plate. The launcher was then subjected to vibration frequencies from 20 to 2000 $\mathrm{Hz}$ for two minutes on each axis $(x, y$, and $z)$, which simulates the worst-case environment for a satellite inside the NanoRacks launch configuration. Between each axis of vibration testing, a sine sweep test was conducted to detect the resonance frequencies of the satellite. Then, the satellite was removed from the launcher, inspected for damage, and re-inserted into the launcher. Photographs of the satellite were taken after each axis of vibration testing and a post-vibration functional test of the D3 deployers was performed.

\section{Results}

The D3 satellite survived vibration testing with no obvious damage when shaken at 9.6 times the force of gravity. There was, however, some shifting of parts that caused one of the deployers to not work as desired. After random vibration testing along the $z$-axis of the launcher, which was the very first test, Deployer 1's motor set screw had loosened (Figure 8). The motor shaft of 
the deployer also slightly moved inward, possibly due to the other screws holding the motor in place coming loose. After the second random vibration test, which was along the $y$-axis, the motor shaft shifted even more inward (Figure 9). During the post-vibration functional test, Deployer 1's motor still worked, but its drum no longer spun due to the set screw loosening, which prevented its boom from deploying. The motors on Deployers 2, 3, and 4 worked nominally, but Deployer 3's set screw also became dislodged from its motor shaft. The screws holding Deployer 3's boom to its drum also self-tightened during vibration testing, causing the boom to jam, but it is unclear when during vibration testing it occurred.

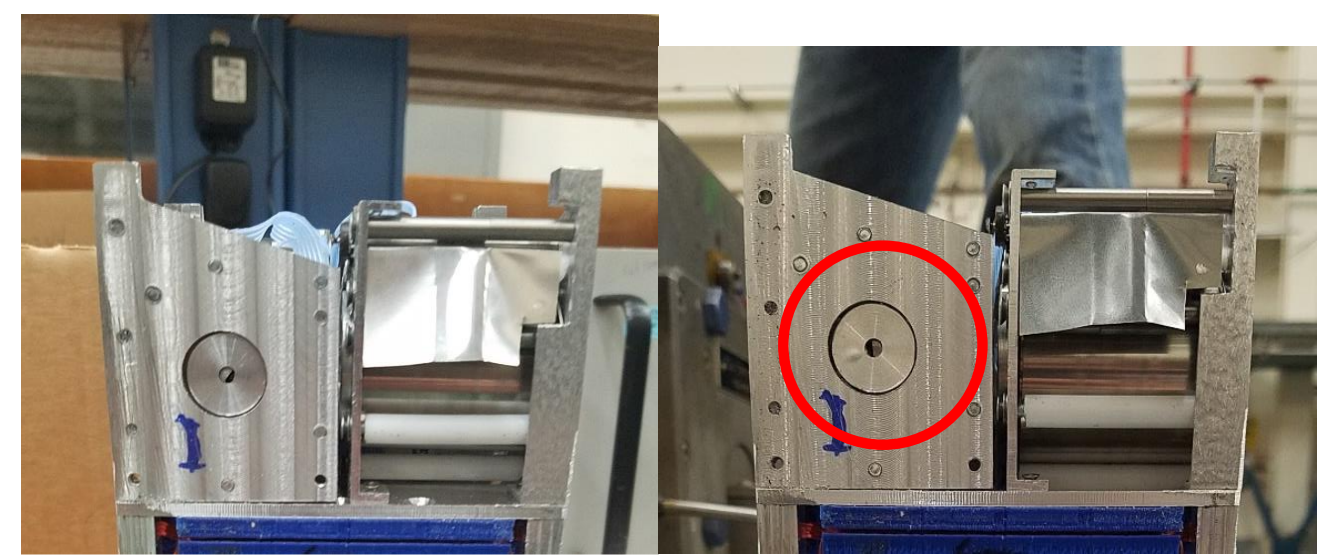

Figure 8. Before (left) and after (right) vibration testing along the $z$-axis of the launcher. Indentation of the drum (circled) caused by the set screw moving is shown on Deployer 1.

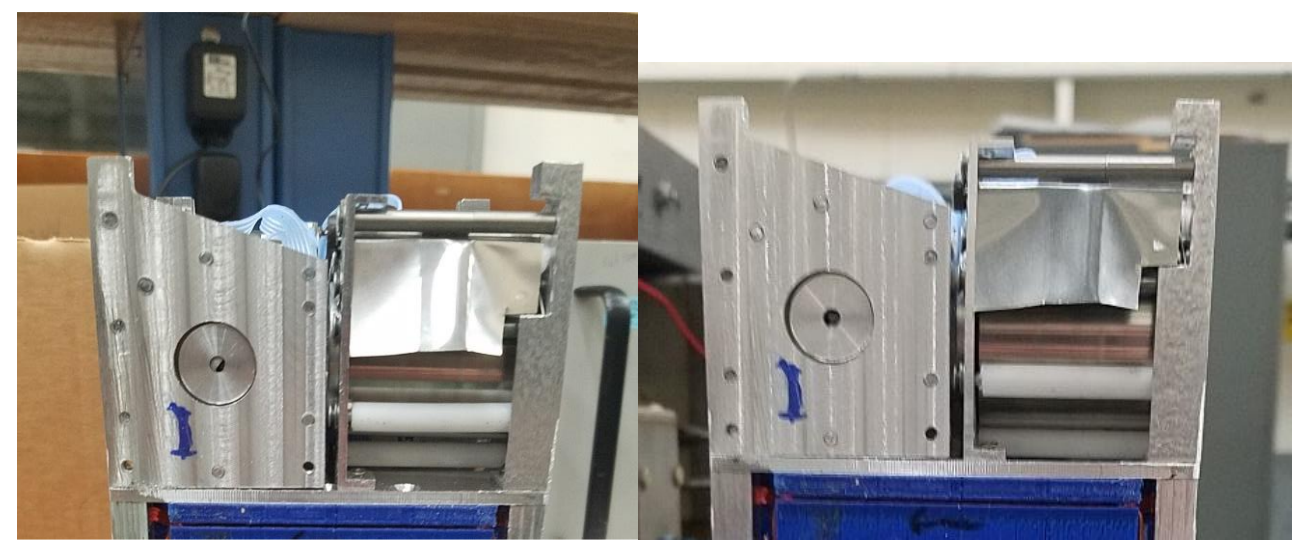

Figure 9. Before (left) and after (right) vibration testing along the y-axis of the launcher. The motor shaft of Deployer 1 has slid back, losing contact with the set screw and making the motor unable to spin.

An accelerometer that was attached to the launcher during testing provided graphical results of the vibration testing. An example of one of the sine sweep graphs is shown in Figure 10 for the $z$ axis. For each axis tested, sine sweep vibration testing showed that there were no resonance 
frequencies below $100 \mathrm{~Hz}$, or no upward "spikes" in the graphs below $100 \mathrm{~Hz}$. This means that the shaking stayed at high frequency bands, which is less likely to cause damage to the satellite.

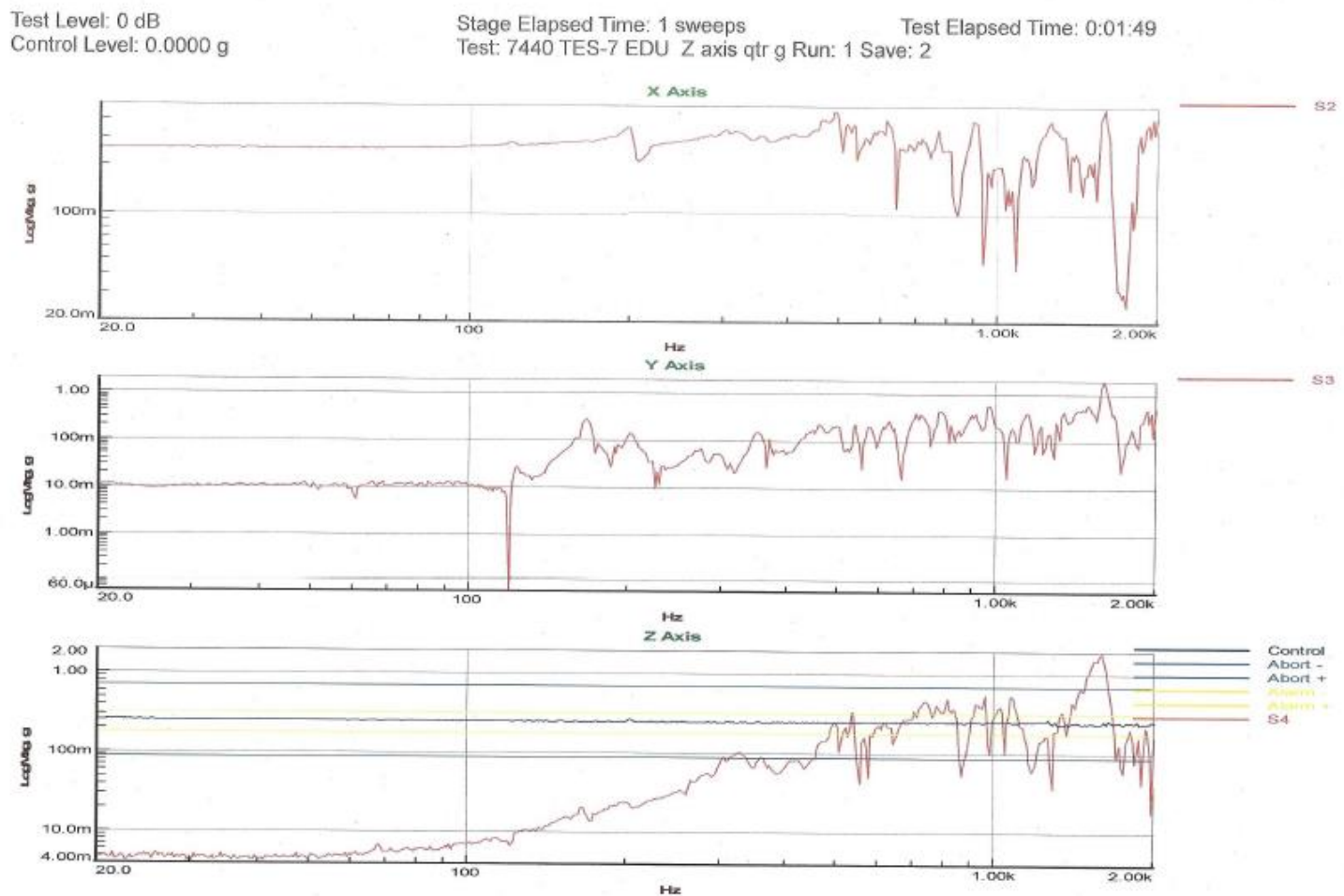

Figure 10. Accelerometer output results of sine sweep vibration testing on the $z$-axis (long axis) of the launcher.

\section{Discussion}

The results from vibration testing confirm that the design of the drag device is sound and that no major changes need to be applied before manufacturing a final drag device. Only slight changes or practices need to be made for the final, flight-ready version of the D3. To ensure that the deployer screws do not self-tighten or self-loosen, liquid thread locker will need to be applied to all screws instead of just the external screws. When the screws are over-tightened, they can scrape against the motor sleeve and jam the deployer. To fix the self-tightening issue, the holes in the drum will be remade without countersinks so that the screws holding the boom can never over-tighten. The drag device's outermost profile that comes into contact with the deployer will need to have tighter manufacturing tolerances in the final iteration. The motors' D-shaped shafts will also need to be milled deeper so that they do not lose contact with their set screws. Other adjustments include using a stronger glue to hold the encoder wheels to their steel shafts so they do not come loose. 


\section{Conclusion}

The results from experimentation and testing determined that further iterations of the D3 device will not need to be made. The results of the vibration testing showed that several screws were susceptible to loosening in the design, but no major damage to the satellite occurred. Thus, the D3 design proves to feasibly endure the simulated conditions of launch, and combined with the guidance and control algorithms and electronic systems in place for the D3 satellite, it will become a reliable way to de-orbit CubeSats from Low-Earth Orbit.

\section{Future Work}

Future work for the ADAMUS lab includes writing flight software and the design and fabrication of the D3 flight control printed circuit board, which will control the D3 motors. After the board is completed, it will be connected with the remaining avionics boards which are to be purchased from vendors. The goal is to launch the D3 CubeSat by 2021. If the mission is successful., the D3 device as well as the guidance and control algorithms involved will demonstrate an effective way to de-orbit CubeSats without propellant and to hopefully mitigate the orbital debris issue for the future.

\section{References}

Cotton, B. (2017). On-Orbit Results from CanX-7 Drag Sail De-Orbit Mission, presented at Proceedings of the 31st Annual AIAA/USU Conference on Small Satellites, Logan, Utah, 2017.

The CubeSat Program. (2014). CubeSat Design Specification Rev. 13. California Polytechnic State University. Retrieved from http://cubesat.org.www.cubesat.org/images/developers/cds_rev13_final2.pdf

Guglielmo, D., Omar, S., Bevilacqua, R., Fineberg, L., Treptow, J., Poffenberger, B., \& Johnson, Y.

(2019). Drag deorbit device: a new standard reentry actuator for cubesats. Journal of Spacecraft and Rockets, 56(1), 129-145. doi:10.2514/1.a34218

Harkness, P., Mcrobb, M., Lützkendorf, P., Milligan, R., Feeney, A., \& Clark, C. (2014). Development status of AEOLDOS - A deorbit module for small satellites. Advances in Space Research, 54(1), 82-91. doi:10.1016/j.asr.2014.03.022

Johnson, N. (2007). The Disposal of Spacecraft and Launch Vehicle Stages in Low Earth Orbit. Retrieved from https://ntrs.nasa.gov/archive/nasa/casi.ntrs.nasa.gov/20070021588.pdf

Murbach, M.S., Papadopoulos, P., Glass, C., Dwyer-Cianciolo, A., Powell, R.W., Dutta, S., GuarnerosLuna, A., Tanner, F.A., \& Dono, A. (2016). Modeling the Exo-Brake and the Development of Strategies for De-Orbit Drag Modulation, presented at 2016 International Planetary Probe Workshop, Laurel, Maryland, 2016. 
NASA. (2012). Process for Limiting Orbital Debris (NASA-STD-8719.14A).

NASA. (2013). General Environmental Verification Standard (GSFC-STD-7000).

Omar, S., \& Bevilacqua, R. (2018). Guidance, navigation, and control solutions for spacecraft re-entry point targeting using aerodynamic drag. Acta Astronautica. doi:10.1016/j.actaastro.2018.10.016 